Lutz Felbick

\title{
Der Compositor extemporaneus Beethoven als »Enkelschüler« Johann Sebastian Bachs
}

1. Heinrich von Kleist: Vom Wert des Extemporierens bei der freien Rede Heinrich von Kleist schwärmt in einem I805 verfassten Aufsatz mit dem Titel Über die allmähliche Verfertigung der Gedanken beim Reden für das Extemporieren bei der freien Rede. Die »Erregung des Gemüts«, die der Redner im Dialog mit seinem Publikum erleben könne, beflügele ihn, ungeahnte Gedanken zu formulieren. Ein solches Reden sei lautes Denken:

\begin{abstract}
»Wenn Du etwas wissen willst und es durch Meditation nicht finden kannst, so rathe ich Dir, mein lieber, sinnreicher Freund, mit dem nächsten Bekannten, der Dir aufstößt, darüber zu sprechen. Es braucht nicht eben ein scharfdenkender Kopf zu sein, auch meine ich es nicht so, als ob Du ihn darum befragen solltest, nein! Vielmehr sollst Du es ihm selber allererst erzählen. Ich sehe Dich zwar große Augen machen und mir antworten, man habe Dir in früheren Jahren den Rath gegeben, von nichts zu sprechen, als nur von Dingen, die Du bereits verstehst. Damals aber sprachst Du wahrscheinlich mit dem Vorwitz, Andere,- ich will, daß Du aus der verständigen Absicht sprechest: Dich zu belehren, und so könnten, für verschiedene Fälle verschieden, beide Klugheitsregeln vielleicht gut neben einander bestehen. Der Franzose sagt, l'appétit vient en mangeant, und dieser Erfahrungssatz bleibt wahr, wenn man ihn parodirt und sagt: l'idee vient en parlant. « ${ }^{\mathrm{I}}$
\end{abstract}

Die Gedanken können also beim Reden entstehen. Man wird ergänzen wollen, die Gedanken kämen auch beim Schreiben, beim Musizieren oder in sonstigen Situationen. Aber ist diese Ergänzung gewinnbringend für die Erfahrung, die Kleist mitteilen will? Er betont in seinem kurzen Aufsatz, man könne Reden und Schreiben in psychologischer Hinsicht nicht gleichsetzen. In der dialogisch verstandenen extemporierten Rede spüre der Vortragende die mehr oder weniger große Zuhörenergie seines Publikums. Es läge »ein sonderbarer Quell der Begeisterung für denjenigen, der spricht, in einem menschlichen Antlitz, das ihm gegenübersteht, und ein Blick, der uns einen halb ausgedrückten Gedanken schon als begriffen ankündigt, schenkt uns oft den Ausdruck für die ganz andere Hälfte desselben. ${ }^{2}$

1 Heinrich von Kleist: Ueber die allmähliche Verfertigung der Gedanken beim Reden, in: Nord und Süd 4 (I878), S.3-7, hier S.3. Ursprünglich war wohl geplant, diesen Beitrag entweder in der Zeitschrift Phöbus oder im Morgenblatt für gebildete Stände zu veröffentlichen. Schließlich kam es postum zu einem von Adolf Wilbrandt eingeleiteten Abdruck in der von Paul Lindau herausgegebenen Zeitschrift Nord und Süd. Vgl. Heinrich von Kleist. Werke und Briefe in vier Bänden, hg. von Siegfried Streller, Peter Goldammer, Wolfgang Barthel, Anita Golz und Rudolf Loch, Berlin/ Weimar 1978, Bd.3, S. $722 \mathrm{f}$.

2 Kleist: Ueber die allmähliche Verfertigung der Gedanken beim Reden, S. 4. 
In der Psychologie des Dialogs hat der Mitteilende Zugang zu Ressourcen, die ihm sonst verborgen bleiben, Gedanken, die anders nicht an die Oberfläche träten, einer Resonanzsituation vergleichbar; der lauschende Zuhörer mobilisiert Saiten beim Vortragenden, die sonst nicht zum Klingen kämen.

Im I9. Jahrhundert wurde die hohe Wertschätzung, die man dieser oralen Kultur entgegenbrachte, durch das weitere Vordringen der Schriftkultur in vielen Bereichen zurückgedrängt. So stellt die Verschriftlichung der Märchen durch die Gebrüder Grimm auf der einen Seite zweifellos einen Gewinn dar, hatte aber zur Folge, dass frei erzählte Märchen immer mehr durch abgelesene Märchentexte verdrängt wurden. Offensichtlich betrachtet dies auch Heinrich von Kleist als einen Verlust und betont Qualitäten, die auf dem Wege der Schriftkultur nicht erreicht werden können: »Etwas ganz Anderes ist es, wenn der Geist schon, vor aller Rede, mit dem Gedanken fertig ist. «

Zweifellos würde ein Interpret klassischer Musik die Wechselwirkung zwischen Vortrag und Publikum ebenso bestätigen wie ein Redner, der seinem Vorlesungskonzept folgt. Aber auf einen feinen, jedoch entscheidenden Unterschied sei hingewiesen: Ein geschickter Redner wird gelegentlich von seinem Vortragsmanuskript abweichen und den Kontakt mit den Zuhörern durch spontan eingebrachte Gedanken suchen. Von den Interpreten klassischer Musikwerke wird diese Abweichung wegen der - aus neuer Zeit stammenden - Forderung nach Werktreue nicht akzeptiert.

In der Mitte des 19. Jahrhunderts entstand eine Priorität des Schriftlichen vor der nicht-schriftlichen Äußerung. ${ }^{4}$ Robert Schumann schrieb am 3. Dezember I838 an Clara Wieck:

»Eines möchte ich Dir rathen, nicht zu viel zu phantasiren; es strömt da zu viel ungenützt ab, was man besser anwenden könnte. Nimm Dir immer vor, alles gleich auf das Papier zu bringen. So sammeln und koncentriren die Gedanken sich mehr und mehr.." 5

In seinen I854 erschienen Musikalischen Haus- und Lebensregeln rät er:»Schreibe also mehr, als du phantasirst. ${ }^{6}$

An der Zunahme der Notendrucke in der ersten Hälfte des I9. Jahrhunderts lässt sich dieser Trend ebenfalls aufzeigen. Die Zeit Beethovens war geprägt vom Übergang der oralen zur Schriftkultur. Die ältere orale Kultur und die nicht-schriftliche Musik-

3 Ebd., S.6.

4 Lutz Felbick: Vom Einfluss der Improvisation auf das mitteleuropäische Musikleben des I9. Jahrhunderts, in: Musiktheorie 20 (2005), S. I65-I8I.

5 Clara Schumann: Jugendbriefe von Robert Schumann, Leipzig I886, S. 296 (3. Dezember I838).

6 Robert Schumann: Musikalische Haus- und Lebensregeln, in: ders.: Gesammelte Schriften über Musik und Musiker, Leipzig I854, Bd.4, S. 293-304, hier S.303. Im Erstdruck der Regeln in der NZfM I7 (I850), Bd.32, Beilage zu Nr.36 (3. Mai I850) ist diese Regel nicht enthalten. 
tradition werden aber dennoch in der zweiten Hälfte des I9. Jahrhunderts, zum Beispiel bei Franz Liszt, immer noch gepflegt und geschätzt. Bis zum 20. Jahrhundert nahm die Fähigkeit immer mehr ab, im Konzert frei zu fantasieren. Ausgenommen davon sind Jazzmusiker, Organisten und äußerst seltene Ausnahmeerscheinungen der Klassikszene wie die venezolanische Konzertpianistin Gabriela Montero, die die »Kunst ohne Werk« noch in einem hohen Maße beherrschen.7 Der Compositor extemporaneus Beethoven soll nachfolgend im Kontext dieses Paradigmenwechsels betrachtet werden.

2. Der Übergang der oralen Kultur zur Schriftkultur in der Musik des 18. Jahrhunderts Der Musikwissenschaftler Robert Lug verfasste I983 den wegweisenden Aufsatz »Nichtschriftliche Musik«. ${ }^{8}$ Er stellt die Musikgeschichte von den Anfängen bis zum 20. Jahrhundert in einem skizzenhaften Grundriss in ihrem Spannungsverhältnis zwischen Schriftkultur und den oralen europäischen Traditionen dar. Zum Verständnis seines Ansatzes ist eine kurze Darstellung des vor allem im angloamerikanischen Sprachraum entwickelten Forschungsgebietes der »oral tradition « erforderlich. ${ }^{9}$

Die nicht auf Schrift basierende Überlieferung des kulturellen Erbes wurde ursprünglich aus der Perspektive der Literaturwissenschaft erforscht. Es kann gezeigt werden, dass die dort vorgenommene Beschäftigung mit den »Axiomen europäischer Kultur« zu Erkenntnissen führt, die grundlegend für ein umfassendes Kulturverständnis sind, und zu einer neuen historischen Sichtweise herausfordert, die auch die Musikgeschichte betrifft. ${ }^{\text {IO }}$ Karl-Hermann Schäfer stellt unter Verweis auf Eric Havelock fest, dass das Phänomen der Oralität in den Jahren I962/63 mit fünf voneinander unabhängig erschienenen Werken von der Wissenschaft aufgegriffen wurde. ${ }^{\text {II }}$ Zwischen ${ }_{9} 963$ und I983 seien zu diesem Thema weitere I36 Schriften erschienen. ${ }^{\text {I2 }}$

7 Derek Bailey: Improvisation. Its Nature and Practice in Music, Ashbourne I980, dt. als: Musikalische Improvisation. Kunst ohne Werk, Hofheim I987.

8 Robert Lug: Nichtschriftliche Musik, in: Schrift und Gedächtnis. Beiträge zur Archäologie der literarischen Kommunikation, hg. von Aleida Assmann, Jan Assmann und Christof Hardmeier, München I983 (Beiträge zur Archäologie der literarischen Kommunikation, Bd. I), S. 245-264.

9 Vgl. Forschungen und Veröffentlichungen des Center for Studies in Oral Tradition, University of Missouri, www.oraltradition.org (29. Juli 2018).

10 Aleida Assmann/Jan Assmann: Schrift und Gedächtnis, in: Schrift und Gedächtnis, S. 265-284, hier S. 265 .

11 Karl-Hermann Schäfer: Homer und Platon als Gründungsväter der Medienpädagogik, in: Franz Fischer Jahrbuch I997, hg. von Anne Fischer-Buck, Karl-Heinz Schäfer, Arno Warzel und Detlef Zöllner, Norderstedt I997, S. 75-95.

12 Davon sind die Werke von Walter Ong: Oralität und Literalität, Opladen I987 (orig. Orality and Literacp, London I982) und Assmanns Schrift und Gedächtnis (I983) als Standardwerke zu betrachten. Die vor 1962 erschienene Literatur fällt demgegenüber kaum ins Gewicht, wiewohl immer 
Eigentlich bedarf diese Theorie keiner ausführlichen Belege, denn in ihr spiegelt sich eine allgemeine Erfahrung. Besonders signifikant ist das spürbar bei den "performativen« Unterschieden zwischen einem öffentlich möglicherweise schlecht abgelesenen Text und einem vertraulich geführten Dialog der Interaktion, der jenseits einer verabredeten Tagesordnung stattfindet. Die öffentlich oder privat gesprochene Sprache kennt zwischen diesen beiden extremen Polen der (Klang-)Rede sehr viele Nuancen.

In der Beziehungsgeschichte zwischen der Schriftkultur und der oralen Kultur sind unterschiedlichste Perspektiven zu beobachten. Vielfach wurden die Unterschiede zwischen beiden Kommunikationsformen ignoriert. So ist bei öffentlichen Reden selten ein Problembewusstsein zu beobachten, ob eine Rede wortwörtlich abgelesen oder frei gehalten wird. Offensichtlich hält man es für zweitrangig, auf welche Weise die »Informationen« vermittelt werden.

Auch im Musikleben wurde häufig die Diskrepanz zwischen der Performance und Aufzeichnung von Musik nivelliert - gemeint sind hier nicht nur visuelle Verschriftlichungen von Musik, sondern auch andere Aufzeichnungen auditiver und/oder visueller Ausprägung - oder die dabei zutage tretenden Widersprüche ignoriert. Die Hilflosigkeit, wie mit den beiden Polen der Schriftkultur und der oralen Kultur umzugehen ist, zeigt sich in paradoxen Ausdrücken wie »orale Literatur «, ${ }^{\mathrm{I} 3} »$ Hörbücher« oder »improvisiertes Werk«. ${ }^{14}$ Auch an den Musikhochschulen mit einer Jazzabteilung sind Auseinandersetzungen zwischen der schriftbasierten und der vornehmlich improvisierten Musik bekannt. Grundlegend ist dabei immer noch die Unterscheidung zwischen der sogenannten E- und U-Musik und zwischen jenen Studierenden, die für ihre spätere Tätigkeit in den sogenannten »Kulturorchestern« vorbereitet werden sollen, und jenen, die in einer anderen Tradition stehen. ${ }^{55}$ Die Ernste Musik, die eng mit der Geschichte der »Kulturorchester« verknüpft ist, entstammt im Regelfall der Schrifttradition.

Ängstlich wirkende Abgrenzungsmanöver gegenüber den oralen Kulturen sind deshalb heute im Musikleben vereinzelt immer noch als ein Nachklang einer älteren

wieder Bezug genommen wird auf eine grundlegende Arbeit von Milman Parry und Albert Lord. Diese hatten mit ihren Thesen zur »homerischen Frage« die Forschungen zu den Epen Homers in ein neues Licht gerückt (vgl. Ong: Oralität und Literalität, S. 24f.). Die auf Homers Epen bezogene »oral-formulaic theory of composition« geht davon aus, dass sich eine orale Kultur bei der Überlieferung der Gedächtnisinhalte grundsätzlich von den Methoden einer Schriftkultur unterscheidet. Vgl. John Miles Foley: Oral-formulaic Theory and Research. An Introduction and Annotated Bibliography, New York I985.

13 Ruth Finnegan: Oral Literature in Africa, Oxford I970.

14 Vgl. Andreas Haug: Der Beginn europäischen Komponierens in der Karolingerzeit. Ein Phantombild, in: Die Musikforschung 58 (2005), S. 225-24I, hier S. 239.

15 Vgl. Lutz Felbick: Das »hohe Kulturgut deutscher Musik« und das »Entartete«. Über die Problematik des Kulturorchester-Begriffs, in: Zeitschrift für Kulturmanagement I/2 (20I5), S. 85-II5. 
Historie zu beobachten. In eurozentristischer Verblendung erscheint in der älteren wissenschaftlichen Literatur der Begriff der »präliteralen Gesellschaft« (vormals: Kultur des "primitiven Menschen«) in seiner Abgrenzung zur »zivilisierten Kultur «. ${ }^{\mathrm{I}}$ Hinweise darauf, dass die europäische Kultur durch die besonderen Werte der oralen Kulturen bereichert werden könnte, findet man dort dagegen kaum. Als seltene Ausnahme ist Walter Ong zu nennen, der feststellt, dass sich afrikanisches Epos und das alte griechische Epos gegenseitig erhellen. ${ }^{\mathrm{I}}$

Es ist durchaus gewinnbringend, die Potentiale der oralen Kultur beziehungsweise die Schattenseiten der Schriftkultur zu betrachten. ${ }^{18}$ Ong führt neun Bereiche an, in denen die orale Kultur der Schriftkultur überlegen ist. Die in einer oralen Tradition verwurzelten Personen verfügen beispielsweise über die Fähigkeit, einen längeren Gesang nach einmaligem Hören wiederzugeben. ${ }^{\text {I9 }}$ Aus seiner Musikhochschularbeit kann der Autor bestätigen, dass diese Fähigkeit, die auch mit einer besonderen Kompetenz der »Höranalyse « beziehungsweise der »Gehörbildung« verknüpft ist, bei Studierenden mit grundlegenden improvisatorischen Fähigkeiten normalerweise deutlich höher ist als bei Studierenden, die sich vornehmlich mit Notenmaterial befasst haben. Wenn viele $\mathrm{Mu}$ siker heute nicht mehr nach Gehör improvisieren können, kann man das durchaus als ein grundlegendes Problem betrachten. Wie Derek Bailey kritisch anmerkt, ist dieses Problembewusstsein aber heute kaum noch vorhanden, denn von Instrumentalisten erwartet man normalerweise nicht mehr, dass sie selber Musik schaffen. ${ }^{20}$ Kein geringerer als Plato, dem die mündlich überlieferte Tradition noch sehr vertraut war, schreibt: »Wer denkt, er könne seine Kunst in Geschriebenem hinterlassen, und wer es aufnimmt mit der Meinung, etwas Klares und Zuverlässiges sei aus Geschriebenem zu entnehmen, der ist von reichlicher Einfalt belastet «. ${ }^{2 \mathrm{I}}$ In der Antike wurde der Kunst der Rhetorik neben den Schriftzeugnissen ein hoher akademischer Rang beigemessen. Selbstverständlich unterschied sich dieses öffentliche Reden von manchen »Vorlesungen« der

16 Jack Goody/Ian Watt/Kathleen Gough: Entstehung und Folgen der Schriftkultur, übers. von Friedhelm Herboth, mit einer Einl. von Heinz Schlaffer, Frankfurt a. M. I986, S. I4; siehe auch Franz Boas: The Mind of the Primitive Man, New York I922.

17 Ong: Oralität und Literalität, S. 34; vgl. Lutz Felbick: Improvisation im Kontext oraler europäischer und außereuropäischer Kulturen, in: Kongressbericht des 4. Jahreskongresses der GMTH, I4.-I7. Oktober 2004, Hochschule für Musik Köln (Onlinepublikation unter www.gmth.de/proceedings.aspx), Veröffentlichung i.V.

18 Vgl. Lug: Nichtschriftliche Musik, S. 250.

19 Ong: Oralität und Literalität, S. 65.

20 Bailey: Improvisation, S. I48.

21 Plato: Phaidros 274c-278b, zit. nach: Schrift und Gedächtnis, S. 7-9, hier S. 7. Das Paradoxon dieser schriftlichen Kritik an der Schrift ist auch im vorliegenden Beitrag nicht zu vermeiden. 
heutigen Universitäten. Wer in der Antike nicht in der Lage war, eine freie Rede über ein Thema zu halten, dokumentierte damit seine eigene fachliche Inkompetenz in dieser Thematik.

2.1 Definitionsprobleme des Kompositionsbegriffs Rudolf Frisius kommt zu der Erkenntnis, dass der Erforschung der Improvisation »in der musikwissenschaftlichen Arbeit bis heute weitgehend unbewältigte Probleme « entgegenstehen. ${ }^{22}$ Die Ursachen für diese offensichtlichen Widersprüche zwischen einer primär schriftorientierten Geschichtsschreibung und den schwer zu integrierenden historischen oralen »Fakten«, besser noch: der faktisch stattgefundenen oralen Kultur, liegen im methodischen Verständnis der Musikwissenschaft. Auch der vorliegende Beitrag sieht sich mit dem Problem der spärlichen Quellenlage konfrontiert. Oft kann nur die Betrachtung des Kontextes - der Erforschung prähistorischer Sachverhalte vergleichbar - weiterhelfen.

Zentrales Quellenmaterial der historischen Musikwissenschaft sind - neben zahlreichen anderen Quellen - die im Laufe der Musikgeschichte entstandenen Kompositionen. Im letzten Jahrhundert wurden auch Werke, die »nur« in elektronischer Aufzeichnung vorliegen, als Tonträger-Quellen mit Werkcharakter akzeptiert, etwa elektronische Musik oder afroamerikanische Musik. Später traten Videoclips hinzu. Eine reduzierte Aufzeichnung eines Videoclips in Form einer Notenpartitur würde zu einer Verfälschung der künstlerischen Intention führen. Man sollte erwarten, dass die Diskussionen um die Definition des Kompositionsbegriffs, der die Grundlage dieser Forschung bildet, abgeschlossen sind. Das ist aber keineswegs der Fall. So kann beispielsweise ein von Andreas Haug im Jahre 2005 verfasster Aufsatz mit dem Titel »Der Beginn europäischen Komponierens in der Karolingerzeit« Anlass zu Missverständnissen geben, denn der karolingische Kompositionsbegriff unterscheidet sich grundlegend von dem der letzten Jahrhunderte. Improvisationen, die schriftlich aufgezeichnet werden, gelten heute nach allgemeinem Verständnis als ein Werk. Sofern die Aufzeichnung allerdings elektronisch erfolgt, wird dieses Musikschaffen nicht als ein Werk mit Opuszahl gewertet. Bei der Verwertungsgesellschaft von musikalischen Rechten GEMA wird diese Form

Rudolf Frisius: Bekanntes und Unbekanntes. Probleme der Formanalyse in improvisierter Musik, www. frisius.de/rudolf/texte/tx733.htm (30. Juli 20I8). Gleiches gilt nach Frisius auch für das Fach Höranalyse; vgl. Lutz Felbick: Das methodisch-didaktische Konzept des argentinischen Lehrbuchs »Analisis Auditivo de la Musica« im Vergleich zu europäischen Höranalyse-Werken, in: Musiktheorie und Vermittlung. Didaktik, Ästhetik, Satzlehre, Analyse, Improvisation, hg. von Ralf Kubicek, Hildesheim $20{ }_{4}$ (Paraphrasen. Weimarer Beiträge zur Musiktheorie, Schriftenreihe der Hochschule für Musik Franz Liszt Weimar, Bd. 2), S. 20I-2I8; vgl. zudem Rudolf Frisius: Fixiertes und nicht Fixiertes. Diesseits und jenseits des Klischees?, www.frisius.de/rudolf/texte/tx734.htm (30. Juli 20I8). 
der Werkaufzeichnung im Regelfall niedriger eingestuft als die gleiche Musik, die in schriftlicher Form vorliegt.

Sehr aufschlussreich sind die diesbezüglichen, differierenden Darstellungen zum Begriff »Komposition « in den beiden musikwissenschaftlichen Standardwerken Musik in Geschichte und Gegenwart (2. Auflage) und im Handwörterbuch der musikalischen Terminologie. Klaus-Jürgen Sachs verschließt sich in seinem MGG-Artikel einer historisch-terminologischen Sichtweise und möchte den Begriff Komposition nur »in allgemeiner (neuzeitlicher) Bedeutung « sehen. Nachfolgend definiert er die Kriterien eines solchen Begriffsverständnisses. Daraus folgert er: »In diesem Sinne nicht eingeschlossen in die Erörterungen des folgenden Artikels wird die Musik solcher oraler Traditionen, für die die genannten Kriterien inadäquat wären. $\ll^{23}$ Warum Sachs den Begriff »Komposition« nicht in den Differenzierungen seiner eigenen Terminologiegeschichte historisch adäquat darstellt, macht er nicht deutlich. ${ }^{24}$ Damit steht er einerseits in Konflikt mit dem Grundkonzept des Lexikons, das sich dadurch auszeichnet, die lexikalischen Begriffe nicht nur aus der Perspektive der Gegenwart, sondern auch in ihrer geschichtlichen Dimension zu betrachten. Andererseits gerät Sachs in seinem eigenen Artikel in einen terminologischen Konflikt und muss bei der Erörterung der mittelalterlichen Musik von einem »vorterminologischen Sinn « des Begriffs compositio sprechen. ${ }^{25}$

Markus Bandur überzeugt in seinen Beiträgen des HmT zum Kompositions- und Improvisationsbegriff mit seiner differenzierteren Darstellung:

„Daß dieses Verständnis [der Komposition als schriftlichem Werk] noch für das frühe I9. Jh. keineswegs als selbstverständlich vorausgesetzt werden kann, machen die nachdrücklichen Betonungen dieser Bedeutung im einschlägigen lexikalischen Schrifttum deutlich. $\ll^{26}$

Bandur charakterisiert an anderer Stelle - Handschin zitierend - »die Eigenart der musikalischen Stegreifproduktion als >Komponieren, das statt auf dem schriftlichen, auf dem >mündlichen Wege erfolgt‘. ${ }^{27}$ Der Autor liefert zu seinem historischen Verständnis des Kompositionsbegriffs in beiden Artikeln zahlreiche Belege, die selbstverständlich

23 Klaus-Jürgen Sachs/Peter Cahn/Rudolf Kelterborn/Helmut Rösing: Art. »Komposition«, in: MGG2, Sachteil, Bd.5, Kassel u. a. I996, Sp.505-557, hier Sp.506.

24 Es verwundert in diesem Zusammenhang, dass im entsprechenden, von Ernest T. Ferand verfassten Artikel in der älteren Ausgabe der MGG die »Wandlungen des Kompositionsbegriffes« ausführlich thematisiert und insofern das Thema aus einem umfassenderen Blickwinkel betrachtet wird (MGG I958, Bd. 7, Sp. I423-I444, hier Sp. I427).

25 Sachs: Art. »Komposition«, Sp.508.

26 Markus Bandur: Art. »Compositio/Komposition«, in: HmT (I996), S.3.

27 Markus Bandur: Art. »Improvisation/Extempore/Impromtu«, in: HmT (2002), S. 7; das Handschin-Zitat stammt aus Jacques Handschin: Über das Improvisieren (I926), in: Gedenkschrift Jacques Handschin. Aufsätze und Bibliographie, hg. von Hans Oesch, Bern I957, S. 327-33I, hier S.327. 
ergänzt werden könnten durch weitere Quellen, wie Äußerungen von Komponisten zur Improvisation aus dem 20. Jahrhundert (beispielsweise von Arnold Schönberg). ${ }^{28}$ Die im $\mathrm{HmT}$ von Bandur vorgenommene Integration der im MGG-Artikel ausgeschlossenen »oralen Tradition« wird der Erörterung des Kompositionsbegriffs historisch gerecht und führt zu einem adäquaten Geschichtsbild.

Beethovens Verwurzelung in dieser Art von Kompositionstradition, wie sie im I8. Jahrhundert gängig war, muss bei einer Betrachtung seines Schaffensprozesses berücksichtigt werden. Der Begriff Composition bezog sich, vereinzelt noch bis ins I9. Jahrhundert, sowohl auf das schriftlose als auch auf das schriftlich fixierte Schaffen. Das Begriffsverständnis von Composition blieb bis ins I8. Jahrhundert »vom schriftlichen Aufzeichnen losgelöst und schließt ganz im Gegenteil auch spontane Ausführung nicht aus. $\ll^{29}$

Eine Auswahl an musiktheoretischen Werken des r8. Jahrhunderts wurde für die hiesige Untersuchung hinsichtlich dieser Fragestellungen beispielhaft ausgewertet. So ergibt sich ein anschauliches Bild des musikgeschichtlichen Kontextes, in den Beethoven im Rahmen seiner Bonner Organistenausbildung hineinwuchs. In dieser Beleuchtung des Kontextes wird absichtlich nicht auf die unmittelbaren Quellen zu Beethovens Improvisationskunst verwiesen, die an anderer Stelle zusammengetragen wurden. Die Quellen geben Auskunft über das spezielle Verständnis von Composition in dieser Zeit, welches von heutigen Definitionen des Kompositionsbegriffs, wie sie beispielsweise bei der GEMA üblich sind, erheblich abweicht.

2.2 »Obligate Fähigkeiten eines Clavierspielers« Johann Kuhnau (I660-I722) verfasste im Jahre I70o die Schrift Der Musicalische Quack-Salber, die von Andreas Werckmeister in einem Text aus dem gleichen Jahr rezipiert wurde. $3^{30}$ Beide berichten von einer Tradition des Extempore-Spiels, die heute im Konzertbetrieb kaum mehr üblich ist. Im Mittelpunkt stand die souveräne Beherrschung des improvisierten musikalischen Handwerks. Die Terminologie des Clavier-Virtuosen und die des guten Komponisten entsprachen nicht der heutigen, denn diese beiden Begriffe galten als identisch. Dieser Berufsstand zeichnete sich durch die Beherrschung des Handwerks aus, selbständig ein

Vgl. Herbert Schramowski: Der Einfluß der instrumentalen Improvisation auf den künstlerischen Entwicklungsgang des Komponisten, Leipzig I968 und Felbick: Vom Einfluss, S. I77.

29 Bandur: Art. »Compositio/Komposition«, S. 20.

30 Andreas Werckmeister: Cribrum musicum oder musicalisches Sieb / darinnen einige Mängel eines halb gelehrten Componisten vorgestellet / und das Böse von dem Guten gleichsam ausgesiebet und abgesondert worden, Quedlinburg/Leipzig I7oo; vgl. Lutz Felbick: Lorenz Christoph Mizler de Kolof - Schüler Bachs und pythagoreischer »Apostel der Wolffischen Philosophie«, Hildesheim 2012 (Hochschule für Musik und Theater »Felix Mendelssohn Bartholdy« Leipzig - Schriften, Bd. 5), S. 86-88. 
Musikstück aus verschiedenen Claves extempore zusammenzustellen. Die Notation der musikalischen Gedanken durch den Komponisten war demgegenüber zweitrangig. Die Priorität lag im Sinne einer primär oralen Kultur eindeutig bei der nicht-schriftlichen Produktion. An der Fähigkeit der Improvisation (Extempore), nicht am Tempo des Notenabsolvierens, erkenne man, so Kuhnau, einen wahren Virtuosen, respektive einen guten Komponisten. Demzufolge beschrieb er den wahren Virtuosen als einen Künstler, der in der Lage sei, eine »ex tempore componierte vollstimmige Sinfonia oder Suonata zu spielen. Ein Musiker hingegen, der die Kunst des Improvisierens nicht beherrsche, sei ein »musikalischer Quacksalber «.3

Wie konnte es dazu kommen, dass man am Anfang des I8. Jahrhunderts einem Musiker, der nicht improvisieren konnte, jegliche Berufsberechtigung als Musiker selbstverständlich auch als Komponist - absprach und sich diese Auffassung in der Mitte des I9. Jahrhunderts grundlegend wandelte? Und vor allem: Welche Position nahm Beethoven in diesem Prozess des steten Wandels ein? Dazu seien die Quellen des 18. Jahrhunderts weiter befragt.

Jeder gute Clavierspieler ist nach Werckmeister im ursprünglichen Sinne des Wortes ein Componist, der weiß »wie die Claves mit einander klingen«. Deshalb zeichne er sich dadurch aus, dass er die »Natur des Claviers [...] inne hat «.32 Ob er ebenfalls fähig ist, seine Clavierkunst schriftlich zu dokumentieren oder Notationen von anderen Musikern zu spielen, ist zweitrangig. Die heute geläufige Trennung zwischen dem Komponisten und dem Interpreten war dem Musikdenken Werckmeisters fremd. Für ihn ist die Fähigkeit eines Clavieristen, »Clavier und Composition« zu beherrschen, identisch mit der handwerklichen Beherrschung der Improvisation (extempore). ${ }^{33}$ Auch an der Fähigkeit des Transponierens erkenne man, »ob ein Organiste sein Clavier im Kopfe hat.«34 Wer »Tabulaturen« (gemeint ist die Notenschrift) von anderen Komponisten abspiele, setze sich dem Verdacht aus, ein Betrüger zu sein. Die Zuhörer würden dadurch »hinter das

31 Johann Kuhnau: Der Musicalische Quack-Salber, nicht alleine denen verständigen Liebhabern der Music, sondern auch allen andern, welche in dieser Kunst keine sonderbare Wissenschaft haben, Dresden I7oo, S. 507 .

32 Andreas Werckmeister: Harmonologia musica oder kurtze Anleitung zur musicalischen Composition [...], Quedlinburg I702, S. 67-69 ([ I26 bzw. I30). Der Begriff »Clavierspieler« beziehungsweise »Clavierist« weicht ebenso wie die Begriffe »Componist« und »Claves« von der heutigen Bedeutung ab und schließt nach damaligem Verständnis alle Musiker ein, die eine »Claviatur beherrschen«: Organisten, Cembalisten et cetera. Die folgenden Quellen zu Werckmeister sind online veröffentlicht: www.felbick.de/diverses (II. Februar 20I8).

33 Andreas Werckmeister: Die nothwendigsten Anmerckungen und Regeln, wie der Bassus continuus oder General-Bass wol könne tractiret werden [...], Quedlinburg I698, Nachdruck hg. von Eitelfriedrich Thom, Blankenburg I985, S. 44 (\$75). 
Licht geführet«, da der angebliche Kenner von »Clavier und Composition« nur die musikalischen Gedanken von anderen - quasi als Plagiat - nachspiele. Deswegen sei es nötig, dass man sich »ein wenig vorsehen / und nicht jedem Praler [sic] alsobald glauben möchte: Denn viele bilden sich ein / sie wissen schon alles«.35 Wer nicht improvisieren kann, bleibe immer »an der Tabulatur hangen / stümpert so was hin / und kömmt nicht weiter. $3^{6}$

Die konkreten Anforderungen an einen Clavier-Spieler gliedern sich nach Werckmeister - neben der allgemeinen musikalischen Kenntnis - in folgende Bereiche:37

A. Obligate Fähigkeiten des Clavier-Spielers (extempore)

- das Spiel nach Akkordsymbolen (»General-Baß«)

- die ad-hoc-Transpositionen (»Transpositionibus«)

- das Spiel von Kadenzen mit richtigen Klauseln (»Clausulieren«)

- das Ex-Tempore-Spielen eines homophonen Satzes zu einem Lied oder Choral (»Contrapunctum simplicem«)

- Lied- und Choralvariation (»Ein Thema anzubringen und zu variiren«)

- Fugenimprovisation (»fugam tractiren«)

- Melodieimprovisation (»ein Thema formiren«)

B. Optionale Fähigkeiten des Clavierspielers

- die Kenntnisse der Notenschrift (»Tabulatur«)

- das Spiel von schriftlich verfassten Musikwerken, damit man »sich dieselben [für das als bedeutender eingestufte Extempore-Spiel] zu Nutze machen« kann.

Die hier genannten Anforderungen an einen Clavierspieler übernahm Lorenz Christoph Mizler 35 Jahre später und sprach vom Virtuosen als einem Musiker, der nach dem Gehör improvisiert und sich zusätzlich für die Fragen der Musiktheorie interessiert. ${ }^{8}$ Der in

35 Werckmeister: Erweiterte und verbesserte Orgel-Probe [...], Quedlinburg 1698, Nachdruck Hildesheim I970, S. 76-78; vgl. auch Werckmeister: Harmonologia, S. 68 (ఏ I28).

36 Werckmeister: Harmonologia, S. 69 ( ( $\mathrm{I}$ I I $)$.

37 Die Fähigkeiten der Clavierspieler wurden bei den Bewerbungsverfahren (»Organistenproben«) geprüft. Schäfertöns nennt in seinem Beitrag weitere Quellen, ohne jedoch Werckmeister zu erwähnen: Reinhard Schäfertöns: Die Organistenprobe. Ein Beitrag zur Geschichte der Orgelmusik im I7. und I8. Jahrhundert, in: Die Musikforschung (1996), S. I42-I52. Auch aus der »Organistenprobe Stockholm I6I3« aus Adriano Banchieris L'organo suonarino (Ricciardo Amadino, Venedig I605) und aus Hans Buchners Fundamentbuch (I506) geht hervor, dass polyphones Stegreifspiel für Clavierspieler selbstverständlich war. Die folgenden Zitate siehe Werckmeister: General-Bass, S. 60 ([I23) und ders.: Harmonologia, S. 66-69 ([ I23-130) bzw. unpag. Register.

38 Lorenz Christoph Mizler: Musikalische Bibliothek, Leipzig I739, Bd. I, 2. Teil (I737), S. 56 f.: »[...] ich habe aber Leute angetroffen, die vor Virtuosen gelten können, auch nicht ungeschickt setzen, nichts destoweniger eine solche Kleinigkeit [von den drei Hauptklauseln in Dur und den drei 
der Barockzeit favorisierte Musikertyp ähnelt demzufolge kaum dem eines heutigen Konzertpianisten oder Cembalisten, der unter dem Aspekt der historischen Aufführungspraxis versucht, sich dem vorliegenden Notentext adäquat anzunähern. Die Quellen liefern vielmehr Hinweise darauf, dass die Arbeitsweise und das Musikverständnis eines Musikers der Barockzeit eher denen eines Jazzmusikers ähneln, dessen Schwerpunkt auf dem Studium der Improvisation liegt. Dies zeigt sich besonders anschaulich an der Biografie Johann Sebastian Bachs.

In seiner Musicalischen Handleitung nennt Friedrich Erhard Niedt den Generalbass den »Anfang zum Componiren«.39 In der Abschrift, in der sich der Autor Carl August Thieme auf Bach beruft, erscheint der Begriff Composition extemporanea und bestätigt die ursprüngliche Einheit dieser heute oftmals getrennten musikalischen Produktionsmethoden: ${ }^{40}$

"Die Trias Harmonica hat eigentlich ihren usum in der Composition weil eben der General Bass ein Anfang ist zum componiren ja würcklich wegen zusammen Stimmung der Con und Dissonantien eine Composition extemporanea mag genennet werden welch derjenige macht so den General Bass schlägt so soll auch dieses Orts hievon meldung geschehen. Wann sich im übrigen ein Lehrbegieriger solchen wohl einbildet und ins Gedächtniß präget so darf er versichert seyn das er schon ein großes Theil der gantzen Kunst begriffen habe. «I $^{\mathrm{I}}$

Bachs improvisatorische Fähigkeiten sind ebenfalls durch einen Bericht über seinen Besuch bei dem berühmten Organisten Johann Adam Reincken überliefert. Dieser hatte fast sechzig Jahre das Organistenamt von St. Katharina in Hamburg inne und war selbst ein angesehener Improvisator. Peter Dirksen stellt fest, dass von Reincken nur wenige Werke bekannt geworden seien und resümiert: »Der Grund dafür dürfte weniger in lückenhafter Überlieferung liegen als vielmehr in der Tatsache, dass Reincken als großer

Hauptklauseln in Moll] nicht gewußt haben. Woher kommet es? weil die meisten practisch MusikVerständigen wenig, oder gar nichts auf das innere Wesen der Musik [im Sinne der Musiktheorie] sehen, und sich darum bekümmern. Sie bleiben bei ihrem Gehör, und sagen: Es kommet alles auf das Naturell an, alle Regeln gelten nichts, wenn man kein gutes Gehör hat.«

Friedrich Erhardt Niedt: Musicalische Handleitung. Gründlicher Unterricht. Erster Theil, Hamburg I7io, Cap. v.

40 Der erste Band von Niedts Lehrwerk befand sich möglicherweise im Besitz Johann Sebastian Bachs, denn er bezieht sich in der ihm zugeschriebenen Generalbasslehre auf diese Handleitung; vgl. die Schrift des Thomanerschülers Carl August Thieme: Des Königlichen Hoff-Compositeurs und Capellmeisters ingleichen Directoris Musices wie auch Cantoris der Thomas-Schule Herrn Johann Sebastian Bach zu Leipzig Vorschriften und Grundsätze zum vierstimmigen spielen des General-Bass oder Accompagnement. Für seine Scholaren in der Music, Leipzig I738, ms. B-Bc 27224 MSM.

41 Vollständig abgedr. in: Philipp Spitta: Johann Sebastian Bach, Bd.2, Leipzig I880, S. 9I2-950, S. 916. 
Improvisator seine Musik wohl nur ausnahmsweise niederschrieb. $^{42}$ Bach bewunderte die Kunst des Orgelmeisters: ${ }^{43}$ Er hätte nicht den Weg nach Hamburg auf sich genommen und seine Werke so häufig bearbeitet, wenn er nicht die überragende »compositorische«Bedeutung Reinckens erkannt hätte. Dieser Komponist verstand es wie kaum ein anderer, die Claves miteinander zum Klingen zu bringen.

Ein frühes Zeugnis zum Unterricht bei Bach ist I7I2 von seinem Weimarer Schüler Philipp David Kräuter überliefert. In seinem Brief an das Scholarchat Augsburg vom 30. April I7ı2 schreibt er über seinen Lehrer:

"[...] er ist ein vortrefflicher, dabey auch sehr getreuer Mann sowohl in der Composition und Clavier, als auch in andern Instrumenten, gibt mir den Tag gewiß 6 Stund zur Information, die ich dann absonderlich zur Composition und Clavier, auch bißweilen zu anderer Instrumenten exercirung hoch vonnöthen habe.«44

Selbstverständlichkeiten der zeitgenössischen Musikpraxis werden in den Quellen oft nur beiläufig erwähnt. Im Falle Bachs enthält dessen Unterrichtsblatt zum Generalbass den Hinweis: »Die übrigen Cautelen, so man adhibiren muß, werden sich durch mündlichen Unterricht besser weder schrifftlich zeigen. «45 Auch den geringen schriftlichen Umfang dieser »Generalbassschule« Bachs kann man als einen Hinweis darauf deuten, dass der Unterricht hauptsächlich mündlich stattfand. Dass die Schüler diese Musik »begreifen« sollten, wurde im ı8. Jahrhundert sehr wörtlich genommen.

Eine anonyme Orgelschule zeigt unter anderem die Gegenbewegung zur Vermeidung von Quintparallelen. Sie wird ganz im Sinne der oralen Kultur haptisch erklärt (»daß die Händ allzeit gegen / oder von einander gehen sollen«). ${ }^{6}$ Heute wird man diese Regel vornehmlich visuell als ein sich schriftlich darstellendes Phänomen erläutern.

42 Pieter Dirksen: Norddeutschland, in: Handbuch Orgelmusik. Komponisten, Werke, Interpretation, hg. von Rudolf Faber und Philip Hartmann, Kassel 2002, S. 6-44, hier S.38.

43 Die Bedeutung Reinckens für die Bach-Forschung wurde im Jahre 2006 nochmals durch den spektakulären Fund einer Musikhandschrift Bachs bestätigt. Dabei handelt es sich um eine Abschrift eines Orgelwerks von Reincken, die um r7oo entstanden ist und zu den frühesten Schriftzeugnissen Bachs zählt.

44 Philipp David Kräuter: Brief an das Scholarchat Augsburg vom 30. April I7ı2, in: Bach-Dokumente, Bd.3: Dokumente zum Nachwirken Johann Sebastian Bachs I750-I80o, vorgel. und erl. von Hans-Joachim Schulze, Leipzig I972, S. 649 (Nr. 53b).

45 Johann Sebastian Bach: Einige höchst nöthige Regeln vom General Basso. di J. S. B. und Einige Reguln vom General Baß (begonnen I725), die als Eintragungen im zweiten KlavierBüchlein der Anna Magdalena Bach überliefert sind, ms. D-B, Mus.ms. Bach P 225, fol. 65 v-67r.

46 Anonymus: Kurzer jedoch gründlicher Wegweiser, vermittelst welches man nicht allein aus dem Grund, die Orgel recht zu schlagen [...], Augsburg I689, S. I8. 
Eine Gemeinsamkeit zwischen Bach und Beethoven bestand in deren Rezeption der Gradus ad Parnassum (I725) von Johann Joseph Fux. ${ }^{77}$ Bach besaß nicht nur das lateinische Original, sondern hatte offensichtlich auch seinen Schüler Mizler angeregt, eine deutsche Übersetzung anzufertigen. Ob das Lehrwerk für Unterricht eher im Sinne der Schriftkultur oder der oralen Kultur genutzt wurde, lässt sich nicht mit Sicherheit entscheiden. Jedenfalls deutet ein Hinweis von Georg Andreas Sorge darauf hin, dass dieses Werk für Übungen zum Erlernen der Improvisation genutzt wurde. Sorge verweist explizit auf Matthesons Kern melodischer Wissenschafften und die Gradus ad Parnassum von Fux. Diese Werke könnten dem Compositor extemporaneus weitere Hinweise im Umgang mit den Dissonanzen geben und seien gute Vorübungen für die Improvisation einer Fuge und die Ausführung eines Themas. ${ }^{48}$

Sowohl Bach als auch Mozart benutzten die schematischen Werterelationen der Kontrapunktgattungen von Fux in ihrem Unterricht. Es ist durchaus vorstellbar, dass die wenigen überlieferten Quellen Hinweise auf diese Praxis des Improvisationsunterrichts liefern. In der Orgelschule Der angehende praktische Organist des Bach-Schülers Johann Christian Kittel wird eine Vorübung zum dem Choral Herr Gott, nun schleuß den Himmel auf in der Relation I:6 gezeigt (Notenbeispiel I), die dann bei Bach in der Bearbeitung des gleichen Chorals in der Relation I: 1:6:3 recht konsequent beibehalten wird (Notenbeispiel 2). 49

Beethoven besaß die 1742 erschienene deutsche Ausgabe der Gradus ad Parnassum. $\mathrm{Ab}$ I8Io unterrichtete er seinen großen Gönner Erzherzog Rudolph in Komposition. Dieser Unterricht wurde wahrscheinlich bereits I809 verabredet, denn ab dem Sommer dieses Jahres kompilierte Beethoven aus den wichtigsten zeitgenössischen Musiklehren Ausschnitte, hauptsächlich zum Kontrapunkt und zur Fugentheorie. Einige dieser Exzerpte legte er in eigens zusammengestellten Heften an. Zum Teil erstellte Beethoven sorgfältige Tabellen, zum Teil fasste er die Kontrapunktlehren im Fließtext mit kommentierten Notenbeispielen zusammen. Ein Heft Beethovens enthält Abschriften zum strengen Kontrapunkt aus dem Lehrwerk von Fux und befasst sich mit der vierten und

47 Fux war nicht der Erste, der Übungen in dieser Art veröffentliche. Johann Nenning legte beispielweise I670 in Bamberg eine Improvisations- beziehungsweise Clavier- und Compositionsschule vor, in der auch Übungen nach dem Muster der späteren Fux'schen Gattungen erscheinen. Spiridion a Monte Carmelo [= Johann Nenning]: Nova instructio pro pulsandis organis, spinettis, manuchordiis etc., I. und 2. Teil, Bamberg I670/7I, im Neudruck hg. von Edoardo Bellotti, Colledara 2003; 3. und 4. Teil [Würzburg ca. I675-I677], im Neudruck nach einer hs. Kopie (Druck nicht erhalten) hg. von Edoardo Bellotti, Latina 2008, S.66f.

48 Georg Andreas Sorge: Vorgemach der musicalischen Composition, Teil III, Lobenstein I747, S. 425.

49 Johann Christian Kittel: Der angehende praktische Organist, Erfurt I8o8, dritte Abtheilung, S. 58 f. 

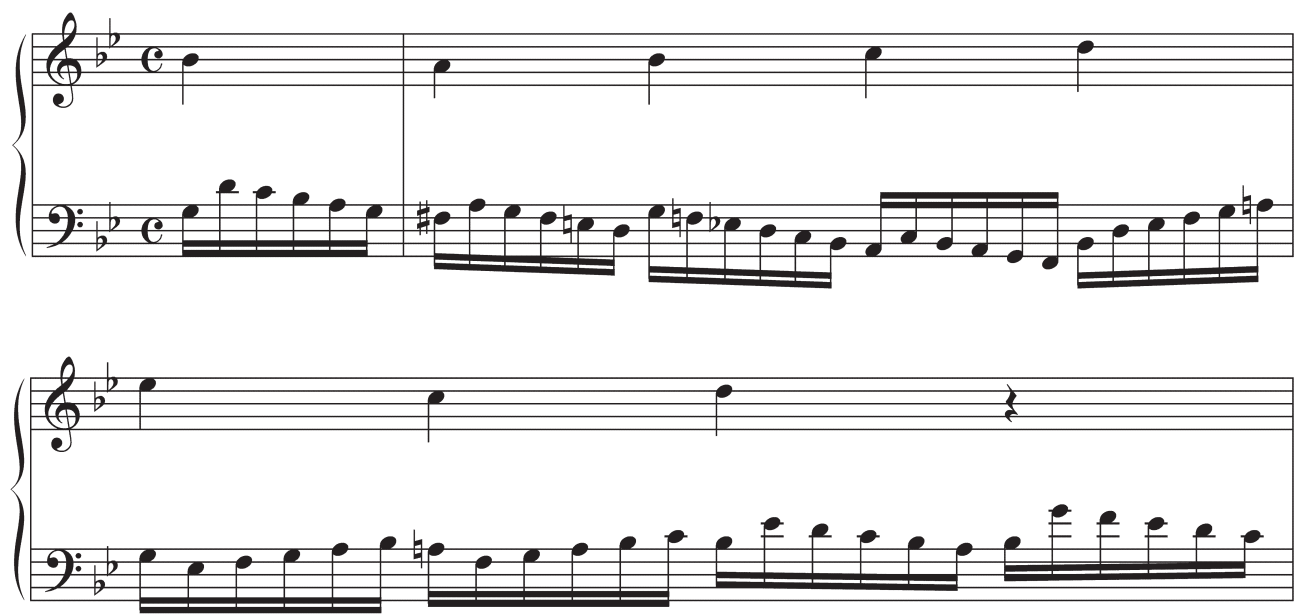

Noten beis PIEL 1 Ausschnitt aus der Choralbearbeitung zu dem Choral Herr Gott, nun schleuß den Himmel auf, verfasst vom Bach-Schüler Johann Christian Kittel. Diese ist als improvisatorische Vorübung im Sinne der Fux'schen Relationen 1:6 zu verstehen.

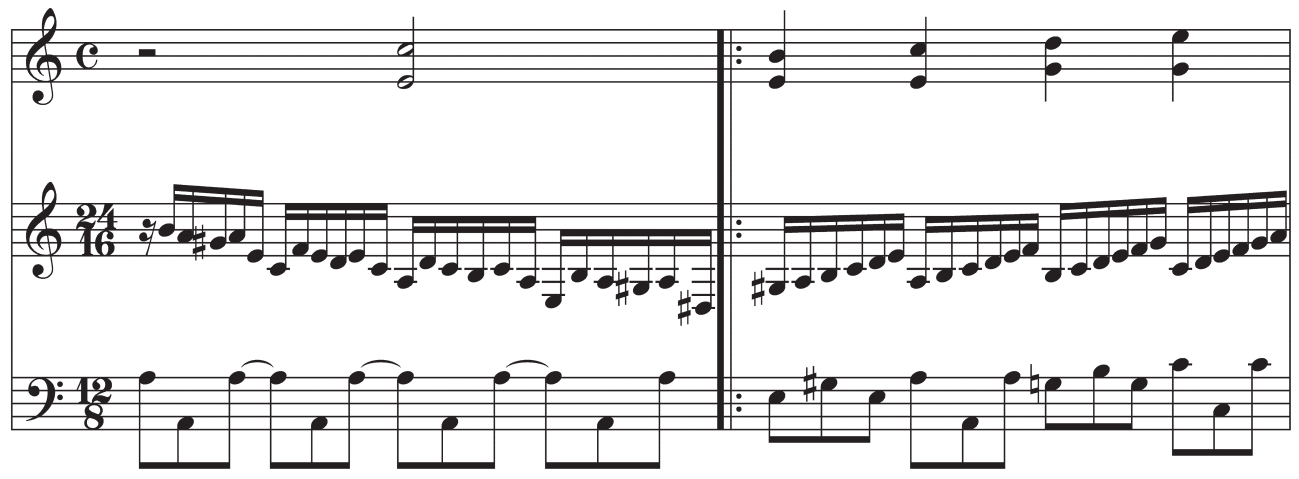

Noten beis PIEl 2 Ausschnitt aus der Choralbearbeitung zu dem Choral Herr Gott, nun schleuß den Himmel auf, ebenfalls basierend auf den oben dargestellten Fux'schen Relationen (Johann Sebastian Bach: Orgelbüchlein, Bwv 617)

fünften Gattung..$^{\circ}$ Es ist angesichts des dargestellten Kontextes höchst unwahrscheinlich, dass der Unterricht primär mittels dieser Aufzeichnungen und notenschriftlicher Übungen stattfand. Ein improvisatorischer Umgang ist als pädagogische Methode eher anzunehmen.

2.3 Allmählicher Bedeutungswandel der Begriffe Komposition und Improvisation In zahlreichen Quellen wird die Nähe oder Gleichsetzung des Improvisations- und Komposi-

50 Vgl. Online-Text zur Abschrift Beethovens: Beethoven-Haus Bonn, Sammlung H. C. Bodmer, нсв Mh $46 \mathrm{f}$. www.beethoven-haus-bonn.de/sixcms/detail.php?id=\&template=dokseite_digita les_archiv_de\&_dokid=ha:wm375\&_seite=I (I2. Februar 20I8). 
tionsbegriffs betont. Mattheson sieht im Extemporieren 1739 einen Vorgang, der identisch sei mit dem »aus dem Stegreiffe componiren $« .5 \mathrm{I}$ Der Titel des vorliegenden Beitrags bezieht sich auf Georg Andreas Sorges Vorgemach der musicalischen Composition (I745), in dem der Begriff Compositor extemporaneus 35 Jahre nach der Erwähnung bei Niedt nochmals erscheint. In seinem 30. Kapitel mit der Überschrift »Wie man etwas gutes und regelmäßiges aus dem Kopffe soll spielen lernen, oder wie man ein Compositor extemporaneus werden könne« führt Sorge eine Anleitung mit I6 Regeln auf, die die Grundprinzipien des Tonsatzes erläutern..$^{2}$

Im Kurtzgefaßten Musicalischen Lexikon, das 1737 bei Johann Christian und Johann David Stößel in Chemnitz erschien, heißt es: »Præambula und Præludia sind solche Vorspiele oder Griffe, die ein Organist ex tempore auf seinem Clavier componiret «.53 Diesem Kompositionsverständnis schließen sich sowohl Carl Philipp Emanuel Bach als auch Jakob Adlung an, denn beide sprechen von der »Composition aus dem Stegereif «.54 Adlung differenziert zwischen den »Papiercomponisten« und den »Claviercomponisten«, die »ihre Sätze und Gänge vorher auf dem Claviere suchen und locken müssen, ehe solche dem Papiere können anvertrauet werden«.55 Die Quellen zur oralen Kultur finden sich vermehrt im I8. Jahrhundert. ${ }^{6}$ Nicht zuletzt spiegelt sich die Betonung der oralen dialogischen Vermittlungsmethode auch in den zahlreichen Unterrichtswerken, die den mündlichen Unterrichtsdialog zwischen Schüler und Lehrer in verschriftlichter Form überliefern beziehungsweise nachahmen. ${ }^{57}$

Das hier ausgeführte Verständnis von nicht-schriftlicher Composition, das sich dann allmählich wandeln sollte, ist aber keinesfalls auf den deutschen Sprachraum begrenzt.

51 Johann Mattheson: Der vollkommene Capellmeister, Hamburg I739, S. I04.

52 Sorge: Vorgemach, S. 4I9-425. Sein Buch mit dem vielversprechenden Titel Anleitung zur Fantasie ist demgegenüber weniger aussagekräftig. Georg Andreas Sorge: Anleitung zur Fantasie, oder zu der schönen Kunst, das Clavier, wie auch andere Instrumente aus dem Kopfe zu spielen; nach theoretischen und practischen Grundsätzen, wie solche die Natur des Klangs lehret [...], Lobenstein I767.

53 Kurtzgefaßtes Musicalisches Lexikon, Chemnitz I737, S. 294.

54 Carl Philipp Emanuel Bach: Versuch über die wahre Art das Clavier zu spielen, Teil I, Berlin I753, S. I3I und Jakob Adlung: Anleitung zu der musikalischen Gelahrtheit, Erfurt I758, S. 736.

55 Adlung: Anleitung, S. 732 und 77I ( (\$ 402); siehe auch Bandur: Art. »Composition«, S. I9.

56 Es wäre eine lohnende Aufgabe, nicht nur diese Schriften, sondern auch alle anderen musiktheoretischen Traktate beziehungsweise die vielen hundert überlieferten Generalbassschulen daraufhin durchzuarbeiten, inwiefern sie direkte oder indirekte Hinweise auf die Beziehungen zwischen Schriftkultur und oraler Kultur enthalten; vgl. die Literaturübersicht bei Jörg-Andreas Bötticher/Jesper B. Christensen: Art. »Generalbass«, in: MGg2, Sachteil, Bd.3, Kassel u.a. I996, Sp. II94-I256 und David A. Damschroder/David Russell Williams: Music Theory from Zarlino to Schenker. A Bibliography and Guide, Stuyvesant, NY I99I.

57 Zum Beispiel Joseph Riepel. Sämtliche Schriften zur Musiktheorie [entstanden I752-I782], hg. von Thomas Emmerich, Wien I996, aber auch Fux und viele andere mehr. 
So nennt Francesco Gasparini 1708 diese Kunst »comporre all'improviso «..$^{8}$ Auch Giovanni Battista Martini spricht I774 von »componevano all' improvviso«.59 Diese Definition ähnelt der lexikalischen des Dizionario e bibliografia della musica von ı826, das Improvisation als »Comporre ed eseguire ex tempore beschreibt. $^{60}$

2.4 Problematik der Notation und die Diskussionen über die »Fantasiermaschine« (1752) Hinweise zur Problematik einer »Paper-Music« liefern auch die Diskussionen um die I752 fertig gestellte »Fantasiermaschine«, über die ihr Erfinder Johann Friedrich Unger I774 berichtet. ${ }^{6 \mathrm{I}}$ Peter Schleuning beschreibt die »Compositionsmaschine «als eine unter einer Claviatur angebrachte Mechanik, durch die »alles, was der Spieler spielt, in währendem Spielen durch einen besonderen Mechanismus in Noten abgedruckt wird«. Diese Notation wurde als Liniennotation realisiert und ähnelt der Darstellung, wie sie heute in der Notationssoftware verwendet wird. Schleuning erwähnt Johann Friedrich Unger und Mr. Creed, die unabhängig voneinander diese Idee einer solchen »Realtime«Papieraufzeichnung von extempore gespielten Fantasien entwickelt hätten. Am I5. März I752 wurde die fertiggestellte Vorrichtung der Berliner Akademie präsentiert, wo sie unter Leonhard Eulers »Directorium ... untersucht und gelobt wurde «. ${ }^{62}$ Unger wirbt für die von ihm erfundene Maschine und beschreibt den Widerwillen mancher Komponisten gegen die Notation: »Sehr oft hat der Componist, zwar Lust zu spielen, aber die Begierde zu schreiben ist desto matter. «3 ${ }^{63}$ Besonders aussagekräftig ist Ungers folgende Passage:

»Ein jeder Componist aber unterscheidet sich von dem andern nicht nur in der Art der Tonfügung überhaupt, sondern es sind auch einem jeden gewisse Auszierungen und Handgriffe eigen, womit er sein Instrument behandelt, und welche der Melodie einen solchen Nachdruck geben, daß sie ohne selbigen nur das halbe Leben behält. Viele davon sind ihm so gewohnt, daß er sich ihrer selbst nicht bewußt ist, indem er sie gebraucht. Und andere sind so zart in einander geschlungen, daß es ihm, wo

58 Francesco Gasparini: L'armonico pratico al cimbalo, Venezia 4I745, S. 59 .

59 Giovanni Battista Martini: Esemplare o sia Saggio fondamentale pratico di contrappunto, Bologna I774, S. 57, Anm.

60 Art. »Improvvisare«, in: Dizionario e bibliografia della musica, hg. von Pietro Lichtenthal, Mailand I826, Bd. I, S.327f., hier S.327.

61 Paper-Music ist der Titel einer CD von Bobby McFerrin. Peter Schleuning: Die Fantasiermaschine. Ein Beitrag zur Geschichte der Stilwende um I750, in: Archiv für Musikwissenschaft 27 (I970), S. I92-2I3.

62 Zit. nach Schleuning: Die Fantasiermaschine, S. I94 und 207. Johann Hohlfeld übernahm die handwerkliche Umsetzung von Ungers Erfindung; vgl. Adlung: Anleitung, S. 577 f.

63 Johann Friedrich Unger: Entwurf einer Maschine wodurch alles was aufdem Clavier gespielet wird, sich von selber in Noten setzt. Im Jahr I752. an die Königl. Akademie der Wissenschaften zu Berlin eingesandt, nebst dem mit dem Herrn Direktor Euler darüber geführten Briefwechsel, und einigen andern diesen Entwurf betreffenden Nachrichten, Braunschweig I774, S.5. 
nicht unmöglich, doch gewiß überaus schwer fallen würde, sie auf eine kennbare Art [in schriftlicher Form] vorzustellen. Die gewöhnlichen Noten sind also nicht geschickt genug, uns diese Schönheiten bekannt zu machen, und wer nicht das Glück hat, den Componisten selbst bey seinem Clavier zu hören, der kann ihn aus seinen Sätzen, auch nicht so genau, als zu wünschen wäre, kennen lernen. $\ll^{64}$

Nicht nur im Zusammenhang mit der Diskussion über die Fantasiermaschine zeigte sich der Widerwille mancher improvisierender Künstler gegen die Aufzeichnung und schriftliche Verbreitung ihrer »Kunst ohne Werk«. Dieses Problem zeigte sich bereits im I7. Jahrhundert. Constantijn Huygens hatte Frobergers letzte Schülerin Sybilla von Württemberg um Noten des Meisters gebeten. Sie antwortete ihm am 23. Oktober I677 ausführlich:

»aber doch mit diser Condition das man sie nit lisse gemein werden, dan er [Froberger] mir offt gesagt das vil van seiner Composition vor ihre Composition ausgeben, und doch nit wisten mit umbzugehen, sondern selbige nuhr verderben und also nit mege das seine sachen under andere Leut hände komen täten«.

Schließlich wisse jeder, »wer die sachen nit von ihme hern Froberger Sel. gelernet, unmüglich mit rechter discretion zu schlagen, wie er sie geschlagen hat «. ${ }^{65}$ Es ist höchst bemerkenswert, dass diese Zeitzeugin über die Unmöglichkeit einer adäquaten Froberger-Interpretation durch nachfolgende Generationen berichtet, und es ist nachzuvollziehen, warum diese zwangsläufig eine Fehlinterpretation sein muss.

Zur Problematik der Notation vermerkt Adlung: »Wie oft sitzt mancher vor dem Claviere, und wünscht bey ausserordentlichen Einfällen, daß das gespielte sogleich auf dem Papiere stehen möchte! Denn ehe man es aufsetzt, ist das beste oft längst vergessen. ${ }^{66}$

$\mathrm{Zu}$ diesem Notationsproblem stellte der Biberacher Organist Justin Heinrich Knecht fest, dass man »den zu Papier gebrachten Phantasien das Gezwungene ziemlich ansiehet. « ${ }^{67}$ Einige Komponisten (zum Beispiel Jean-Henri d'Anglebert) lösten dieses Problem, indem sie nur eine approximative Notationsskizze vorgaben, die den zeitlichen

64 Ebd., S. 4 .

65 Johann Jacob Froberger: Clavier- und Orgelwerke abschriftlicher Überlieferung. Partiten und Partitensätze, hg. von Siegbert Rampe, Kassel 2002 (Neue Ausgabe sämtlicher Werke, Bd.3), S. viıI; vgl. auch Pieter Dirksen: A Froberger Miscellany: r. The Huygens-Sibylla Correspondence (г666I668); 2. A Preliminary Source-List of Froberger's Keyboard Music; 3. Bibliography, in: The Harpsichord and its Repertoire, hg. von Pieter Dirksen, Utrecht I990, S. 325-332.

66 Adlung: Anleitung, S. 577 , Anm.

67 Justin Heinrich Knecht: Etwas über das Präludieren überhaupt, in: Musikalische Realzeitung für das Jahr I788, Bd. I, hg. von Heinrich Philipp Bossler, Speyer I788, Nr. I3 (24. September I788), Sp. 98-Ior, hier Sp. гоo. 
Ablauf nur andeutete. ${ }^{68}$ C.P.E. Bach sträubte sich sogar lange gegen die Notation von Clavierfantasien überhaupt, und fragte sich, »wie viele sind deren, die dergleichen lieben, verstehen und gut spielen? ${ }^{6} 9$

2.5 C. P. E. Bach als bedeutender Lehrmeister des 18. Jahrhunderts Schleuning beschrieb in seinen Forschungen zur Improvisationskultur des I8. und I9. Jahrhunderts nicht nur die Fantasiermaschine, sondern verfasste einen für die hiesige Thematik aufschlussreichen Beitrag mit dem Titel „Carl Philipp Emanuel Bachs berühmtester Schüler-Ludwig van Beethoven «.70 In der Tat kann man wirklich von einer Schülerschaft sprechen, denn Beethoven beispielsweise studierte die Methode des Bachsohns nicht nur sehr intensiv, sondern war davon so überzeugt, dass er wiederum Czerny auf der Grundlage von Bachs Clavierschule ausbildete. ${ }^{\text {II }}$ Im zweiten Band seiner Clavierschule widmete der BachSohn das 4r. Kapitel dem Thema »Von der freyen Fantasie«. Für ihn »war die Improvisationsfähigkeit für den Clavierspieler immer noch obligat«:

»Man verlanget noch überdies, daß ein Clavierspieler Fantasien von allerley Art machen soll; daß er einen aufgegebenen Satz nach den strengsten Regeln der Harmonie und der Melodie aus dem Stegereif durcharbeiten, aus allen Tönen mit gleicher Leichtigkeit spielen, einen Ton in den andern im Augenblick ohne Fehler übersetzen, alles ohne Unterscheid vom Blatte weg spielen soll, es mag für sein Instrument eigentlich gesetzt sein oder nicht; daß er die Wissenschaft des Generalbasses in seiner völligen Gewalt haben, selbigen mit Unterscheid, oft mit Verläugnung, bald mit vielen, bald mit wenigen Stimmen, bald nach der Strenge der Harmonie, bald galant, bald nach einem zu wenig oder zu viel, bald gar nicht und bald sehr falsch bezieferten Basse spielen soll; daß er diesen Generalbaß manchmahl aus Partituren von vielen Linien, bey unbezieferten, oder ofte gar pausirenden Bässen, wenn nemlich eine von den andern Stimmen zum Grunde der Harmonie dienet, ziehen und dadurch die Zusammenstimmung verstärcken soll, und wer weiß alle Forderungen mehr?«

»Besonders aber kan ein Clavieriste vorzüglich auf allerley Art sich der Gemüther seiner Zuhörer durch Fantasien aus dem Kopfe bemeistern [...], welche nicht in auswendig gelernten Passagien oder gestohlnen Gedancken bestehen . $^{2}$

68 Zum Beispiel im Prélude seiner Première Suite, Paris I689, oder auch bei Jean-Philippe Rameau: Premier Livre de Pièces de Clavecin, Paris I706, Prélude.

69 Brief an Johann Nikolaus Forkel vom ro. Februar 1775; vgl. Carl Hermann Bitter: Carl Philipp Emanuel und Wilhelm Friedemann Bach und deren Brüder, Berlin I868, Bd. I, S.340-342, hier S.34I.

70 Peter Schleuning: Carl Philipp Emanuel Bachs berühmtester Schüler - Ludwig van Beethoven, in: Carl Philipp Emanuel Bach als Lehrer. Die Verbreitung der Musik Carl Philipp Emanuel Bachs in England und Skandinavien, hg. von Hans Günter Ottenberg, Frankfurt (Oder) 2005, S. I5I-I66.

71 Marianne Betz: Art. »Fantasia«, in: $\mathrm{HmT}$ (200I), S. I2; vgl. Carl Czerny: Erinnerungen aus meinem Leben, hg. von Walter Kolneder, Straßburg/Baden-Baden I968, S. 28.

72 C. P. E. Bach: Versuch, Teil I, Vorrede [S. 2] und S. I22 f.; siehe auch: Von der freyen Fantasie, ebd., Teil 2, Berlin I762, S. 325-34r. Zu der Formulierung der "gestohlnen Gedancken« vgl. ähnlich lautende Formulierungen bei dem oben bereits zitierten Werckmeister. 
2.6 Markante Gegenpositionen 1733-1752: Die freie Fantasie als »Missgeburt« Im Umfeld des Thomaskantors J. S. Bach wurde zur Zeit des radikalen Rationalismus Wolff'scher Prägung engagiert über den Wert und Unwert des Fantasierens diskutiert. I733 veröffentlichte der Leipziger Universitätslehrer und Philosoph Johann Christoph Gottsched die Schrift Erste Gründe der gesamten Weltweisheit. Sie galt als populäre Zusammenfassung der Philosophie von Christian Wolff, dem Erfinder des »Geistes der Gründlichkeit«, wie Kant den philosophischen Vorgänger charakterisierte. ${ }^{73}$ Diese Gründlichkeit veranlasste Gottsched dazu, sich energisch gegen das Extemporieren auszusprechen:

»Die eine Art sich etwas ohne Beobachtung eines zureichenden Grundes einzubilden, heißt eigentlich träumen, oder phantasiren: Weil man im Schlafe oder hitzigen Fieber dergleichen Einfälle zu haben pflegt. Gleichwohl bedienen sich ungeschickte Mahler, Poeten und Componisten vielmals dieser Kraft, und bringen dadurch lauter Misgeburten zur Welt, die man Träume der Wachenden nennen könnte. Die Grotesken der ersten, und die ungereimten Fabeln der andern können hiervon zu Exempeln dienen. «74

Andere Aufklärer übernahmen diese Gedanken Gottscheds. Beispielsweise vertrat der Bach-Schüler Mizler die Auffassung, ein Musikstück müsse soweit möglich nach der Vernunft eingerichtet werden und der schriftlichen Nachvollziehbarkeit unterliegen. Demzufolge sei ein nicht schriftlich verfasstes, aus dem Stegreif entstandenes Musikstück »nicht viel werth«. Offensichtlich kannte Mizler die oben zitierte Passage seines Lehrers Gottsched, denn er sprach ebenfalls von »Mißgeburthen«, die angeblich durch spontane Kreationen entstünden.75

Gottscheds Kritik an der Stegreifproduktion trat auch in dem sogenannten Hanswurststreit zutage, der sich im Zusammenhang mit seinem Bemühen um ein den Zielen der Aufklärung dienendes zeitgenössisches Theater entfachte. Seit dem I6. Jahrhundert trat dieser »Hans Worst« in Stegreifkomödien auf. Seine Bühnenkunst und seine Wortbeiträge waren spontan improvisiert und konterkarierten in vielen Fällen die Anliegen der Zensur. Hintergrund dieser Problematik war die damals übliche strenge Aufsicht der aufgeführten Bühnenwerke durch die Zensurbehörden. Es ist leicht nachvollziehbar, dass sich die Improvisationen derartigen vorherigen, rationalen Kontrollmechanismen entziehen. Gottsched, dem eine in diesem Sinne kontrollierte Bühnenkunst ein großes Anliegen war, hatte Caroline Friederike Neuber I737 deshalb dazu veranlasst, die Figur

Immanuel Kant: Critik der reinen Vernunft, Riga ${ }^{2}$ I787, Vorrede, S. XxxvI.

74 Johann Christoph Gottsched: Erste Gründe der gesamten Weltweisheit, Leipzig I733, Bd.I, S. 224 ( $(9458)$.

75 Felbick: Lorenz Christoph Mizler, S. 87; Mizler: Musikalischer Staarstecher, Leipzig I740, S. 97. Das Argument Mizlers, jegliche spontane Äußerung widerspreche der Natur, ist irreführend und nicht nachvollziehbar, denn in der »Natur« gab es schon vor der Erfindung der Schrift sprachliche Extempore-Äußerungen. 
des Hans Wurst von der offenen Bühne zu verbannen. Dieser wurde »zum Tode verurteilt und anschließend als Puppe auf einem Scheiterhaufen im Freien demonstrativ verbrannt $« .{ }^{76}$ Das erregte großes Aufsehen und zeigte besonders in Wien Wirkung, denn die Missachtung dieser beliebten Stegreif-Tradition traf auf Widerstand.77 Da die Zensur die vor der Aufführung schriftlich nicht fixierten Gedanken nicht vorab begutachten konnte, ordnete wiederum Maria Theresia 1752 durch ein Extemporierverbot an, dass nur die Vorstellungen, die »wol ausgearbeitet befunden werden, auf dem hiesigen Theater zu producieren gestattet seien . $^{78}$ Derweil kritisierte Gotthold Ephraim Lessing im Jahre I759 die Initiative Gottscheds, denn dieser habe einen »Fluch auf das extemporiren« gelegt.79

Unter den Aufklärern gab es also sehr unterschiedliche Einschätzungen vom Wert der Improvisation. Es gab solche, die die orale Tradition noch sehr zu schätzen wussten. Dem standen die vermeintlich Fortschrittlichen gegenüber, die die Eigengesetzlichkeit und die künstlerischen Potentiale der freien Fantasie verachteten und stattdessen nur die Vorzüge der gründlichen schriftlichen Ausarbeitung sahen.

Wo stand Beethoven? War ihm das »moderne« Musikdenken im Sinne der Gottsched-Tradition oder die konservative Extempore-Tradition näher? Zweifellos bemühte sich auch Beethoven um eine gründliche Ausarbeitung seiner musikalischen Gedanken, aber in seinem positiven Verhältnis zur Improvisation kam eher eine konservative Seite seines Musikdenkens zum Vorschein; eine Art Musik zu schaffen, die ihn ebenso begeisterte wie das differenzierte Ausarbeiten eines Notentextes.

2.7 Gleichzeitigkeit der alten und neuen Denkweise Im Vorbericht von Friedrich Wilhelm Marpurgs Clavierschule Die Kunst das Clavier zu spielen von I76I wird auf die Neuartigkeit seiner Methode hingewiesen. Man solle in dem Werk »nicht mehr suchen, als einen vollständigen Unterricht von einer regelmäßigen und bequemen Fingersetzung.« Sehr bewusst sei die Anweisung zum Generalbass-Spiel nicht in die Clavierschule integriert worden..$^{8 \circ}$ Dieses neue Curriculum, welches sich nun auf die korrekte und physiologisch optimale Ausübung konzentriert, ist kennzeichnend für eine grundlegende

76 Herbert Ziessler: Vom Leben und Wirken der Frau Neuberin, Reichenbach I957, S.3I.

77 Sybille Maurer-Schmoock: Deutsches Theater im I8. Jahrhundert, Tübingen I982, S. I68-I73.

78 Vgl. Karl von Görner: Der Hans Wurst-Streit in Wien und Joseph von Sonnenfels, Konegen I884, S. 4.

79 Gotthold Ephraim Lessing: Briefe, die neueste Literatur betreffend [...], Bd. I, Teil I, Berlin I759, S. 99, Siebzehnter Brief vom I6. Februar I759. Dieser Brief enthält eine grundlegende Kritik an Johann Christoph Gottsched.

80 Friedrich Wilhelm Marpurg: Die Kunst das Clavier zu spielen, Augsburg I76I, unpag. Vorbericht. Die Ausgabe ist ein Augsburger Nachdruck der Berliner Erstausgabe von I751. Das Vorwort stammt nicht vom Verfasser selbst. 
Veränderung. Das Verständnis der harmonischen Abläufe und Stimmführungsregeln bildet nicht mehr den Mittelpunkt des Unterrichts, es wird zu einer entbehrlichen Randerscheinung.

Allerdings folgen nicht alle Zeitgenossen Marpurgs dieser neuen Entwicklung: Offensichtlich unterrichtete Leopold Mozart nicht nach dieser neuen Methode, denn die Vermittlung der kompositorischen Prinzipien war bei ihm zentraler Unterrichtsgegenstand. ${ }^{8 \mathrm{I}}$ Dies zeigt sich bekanntlich bei seinem bekanntesten Schüler, seinem eigenen Sohn Wolfgang Amadeus. In einer Frankfurter Konzertanzeige hieß es I763, dass er »nicht nur auf dem Flügel, sondern auch auf einer Orgel (solange man zuhören will, und als allen, auch den schwersten Tönen [Tonarten], die man ihm benennen kann) vom Kopf phantasiren « könne. ${ }^{82}$ Auch der Lehrer Placidus Scharl bestätigte in seinen Aufzeichnungen der Jahre I759-I770, »musikalisches Fantasieren sei die Passion des jungen Mozart gewesen. ${ }^{83}$ Der Vorgang des Komponierens war für Mozart nicht identisch mit dem Akt des Notenschreibens. Wie vielfach berichtet, entstand die Komposition im Kopf und wurde dann entweder auf dem Clavier improvisiert oder in wesentlich mühevollerer Arbeit zu Papier gebracht. Letzteres gehörte nicht zur Passion Mozarts, sondern war ihm eine lästige Pflicht. Trotz der vielfach nachweisbaren Bedeutung der Improvisation für Mozarts Schaffen sucht man in der heutigen Mozart-Forschung vergeblich nach einer namhaften Institution, die sich dieses Schaffensbereichs mit ähnlicher Sorgfalt annimmt, wie dies in der Internationalen Stiftung Mozarteum mit der Neuen MozartAusgabe geschieht. ${ }^{84}$ Immerhin wird in der Gesamtausgabe auf zeitgenössische Berichte hingewiesen, die ausführen, dass Mozart bereits im Alter von sieben Jahren »bald mit Accorden ganze Stunden aus seinem Kopfe phantasieren « konnte. ${ }^{85}$

Bei der - möglicherweise nur legendären, aber durchaus vorstellbaren - Begegnung Mozarts mit dem jungen Beethoven im Jahre 1787 habe dieser eine Fantasie gespielt. » >Das ist recht hübsch<, sagte Mozart, saber eingelernt<. Gekränkt bat sich Beethoven ein

81 Ulrich Kaiser: Die Notenbücher der Mozarts als Grundlage der Analyse von W. A. Mozarts Kompositionen I76I-I767, Kassel 2007, S. 28 und 84.

82 Anzeige aus dem Intelligenz-Blatt der freien Stadt Frankfurt vom 30. August I763, in: Maria BelliGontard/Johann H. Lerz/Johann Adam Creutz: Leben in Frankfurt am Main. Auszüge der Frag- und Anzeigungs-Nachrichten (des Intelligenz-Blattes) von ihrer Entstehung an im Jahre I722 bis I82I, Frankfurt a. M. 1850, S. 26.

83 Schramowski: Der Einfluß der instrumentalen Improvisation, S. 72; siehe auch Hermann Abert: W. A. Mozart, Bd. I, Leipzig I955, S. 25.

84 Vielfache Belege zur Improvisationspraxis bei Mozart finden sich bei Schramowski: Der Einfluß der instrumentalen Improvisation, S. 72-80.

85 Zitat aus dem Augsburgischen Intelligenz-Zettel vom I9. Mai I763, zit. nach Mozart. Die Dokumente seines Lebens, ges. und erl. von Otto Erich Deutsch, Kassel I96I (Wolfgang Amadeus Mozart. Neue Ausgabe Sämtlicher Werke, Bd.x/34), S. 22; vgl. Kaiser: Die Notenbücher, S. 84 . 
Thema aus und fantasierte so, daß Mozart zu einigen Freunden sagte: `Auf den gebt acht, der wird euch noch was erzählen «.« 86

Diese markanten Fragmente sind exemplarische Zeugnisse der hohen Improvisationskultur der Wiener Klassiker. Beethoven schätzte zwar das musiktheoretische Erbe des I8. Jahrhunderts außerordentlich und beherrschte die Improvisationskultur hervorragend, er hatte aber ebenfalls an musiktheoretischer Literatur Interesse, in welcher die Improvisation als zentrale Fähigkeit des Komponisten und Clavierspielers keine Rolle mehr spielt. So hatte er offensichtlich Johann Philipp Kirnbergers Die Kunst des reinen Satzes in der Musik studiert, denn er kopierte daraus zwei Beispiele, die er wahrscheinlich für den Unterricht nutzte. ${ }^{87}$

Wiewohl man gegen Ende des I8. Jahrhunderts noch nicht von einem Aussterben der oralen Kultur sprechen kann, mehren sich die Quellen, in denen auf das Wagnis der Improvisation hingewiesen wird. So wurde die musikalische Kunst mehr und mehr perfektioniert und Aufführungsrisiken wurden gemieden. So rät etwa Daniel Gottlob Türk ${ }^{7} 89$ in der Klavierschule, bei einer Kadenz doch besser auf die Improvisation zu verzichten und die Gedanken vorher zu notieren. ${ }^{88}$

Andererseits hält Türk an der Verbindung der Klavierschule mit dem Generalbassunterricht fest, denn in seiner I79I erschienenen Kurzen Anweisung zum Generalbaßspielen weist er auf ein bereits entworfenes umfangreicheres Werk über den Generalbass hin, das »als zweyter Theil meiner größern Klavierschule angesehen werden kann.«99

Bis zur Mitte des I9. Jahrhunderts gibt es bei Heinrich Christoph Koch, Johann E. Häuser, Anton Schindler und anderen zwar noch vereinzelt Hinweise, die auf die alte Tradition des Extemporierens hindeuten, nichts desto trotz wird die »Oralität« zunehmend zu einer Randerscheinung des öffentlichen Musiklebens.

Das Studium des Musikdenkens im I8. und I9. Jahrhundert zeigt, dass man Beethoven sowohl als einen Compositor extemporaneus als auch einen vom »Geist der Gründlichkeit« geprägten Tonsetzer begreifen sollte. In diesem Sinne kann er als ein Grenzgänger zwischen dem alten und neuen Kompositionsbegriff und zwischen oraler und schriftlicher Kultur verstanden werden. Die in Umrissen hier vorgelegte Analyse des allmählichen Paradigmenwechsels ermöglichte eine neue Perspektive auf den Schaf-

86 Howard Chandler Robbins Landon: Mozart and Vienna, London I99I, S.36.

87 Beethoven-Haus Bonn, Manuskript Bн 8I, www.beethoven.de/sixcms/detail.php?id=\&template =opac_bibliothek_de\&_opac=hans_de.pl\&_t_show=x\&_reccheck=6I4\&_wert=kunst+des+reinen +satzes\&_wertre g=SSW\&_x=u, (27. Januar 20I8).

88 Daniel Gottlob Türk: Klavierschule oder Anweisung zum Klavierspielen für Lehrer und Lernende, Leipzig/Halle I789, S.3г3.

89 Daniel Gottlob Türk: Kurze Anweisung zum Generalbaßspielen, Leipzig/Halle I79I, unpag. Vorerinnerung, [S. I]. 
fensprozess Beethovens. Der Terminus Composition sollte im Begriffsverständnis des I8. Jahrhunderts beleuchtet werden. Unter diesem Einfluss steht auch Beethoven, bei dem im Gegensatz zu manchen Gelehrten des I8. Jahrhunderts ein positives Verhältnis zum Extemporieren nachweisbar ist. Deshalb ist Beethoven weit von der Meinung Gottscheds und Mizlers entfernt, die »Kunst ohne Werk« sei als eine Missgeburt zu bezeichnen. Aber der Komponist unterscheidet sich in einem wesentlichen Punkt von seinen Vorgängern, denn für den oben erwähnten Organisten Reincken war die Notation seines musikalischen Schaffens recht unbedeutend, Beethoven steht hingegen trotz seiner Verbindung zu den alten oralen improvisatorischen Traditionen auch unter dem Einfluss einer sich im I9. Jahrhundert mehr und mehr entwickelnden Schriftkultur und der damit verbundenen explosionsartig ansteigenden Zahl von Notendrucken, die den Musikverlagen gute Gewinne garantierte. 


\section{Inhalt}

Vorwort 7

Maria Grazia Sità Improvisation and the Rhetoric of Beginning

Lutz Felbick Der Compositor extemporaneus Beethoven als »Enkelschüler« Johann Sebastian Bachs 34

Giorgio Sanguinetti A Partimento in Classical Sonata Form by Giacomo Tritto 57

Michael Lehner »Und nun sehe man, was hieraus gemacht werden kann«. Carl Czernys Anleitung zum Fantasieren als implizite Harmonie- und Formenlehre 69

Leonardo Miucci Completing the Score.

Beethoven and the Viennese Piano Concerto Tradition $\quad 9^{8}$

Martin Skamletz Joseph Preindls Klavierfantasien als Echo von Opern- und Oratorienaufführungen in Wien um I800 II6

Martin Skamletz »Classisches Clavierspiel«. Joseph Lipavsky und das Rondeau-Fantaisie $\quad$ I37

Sonja Wagenbichler Showdown am Klavier. Zur Kultur pianistischer Wettstreite im Wien des I8. und I9. Jahrhunderts $\quad$ I64

Stephan Zirwes Formale Dispositionen in den komponierten Fantasien zur Zeit Beethovens I75

Nathalie Meidhof Variation, »Harmoniekenntniss« und Improvisation. Beethovens Fünf Variationen über das englische Volkslied »Rule Britannia« für Klavier in D-Dur (WoO 79) I92

Namen-, Werk- und Ortsregister 202

Die Autorinnen und Autoren der Beiträge $\quad 208$ 


\section{DAS FLÜCHTIGE WERK}

Pianistische Improvisation der Beethoven-Zeit •

Herausgegeben von Michael Lehner, Nathalie Meidhof

und Leonardo Miucci unter redaktioneller

Mitarbeit von Daniel Allenbach 


\section{MUSIKFORSCHUNG DER \\ Hochschule Der KÜnste Bern \\ Herausgegeben von Martin Skamletz \\ und Thomas Gartmann}

Band 12 
0 Dieses Buch ist in gedruckter Form im Juli 20I9 in erster Auflage in der Edition Argus in Schliengen/Markgräflerland erschienen. Gestaltet und gesetzt wurde es im Verlag aus der Seria und der SeriaSans, die von Martin Majoor im Jahre 2000 gezeichnet wurden. Gedruckt wurde es auf Eos, einem holzfreien, säurefreien, chlorfreien und alterungsbeständigen Werkdruckpapier der Papierfabrik Salzer im niederösterreichischen Sankt Pölten. Das Vorsatzpapier Caribic cherry wurde von Igepa in Hamburg geliefert. Rives Tradition, ein Recyclingpapier mit leichter Filznarbung, das für den Bezug des Umschlags verwendet wurde, stellt die Papierfabrik Arjo Wiggins in Issyles-Moulineaux bei Paris her. Das Kapitalband mit rot-schwarzer Raupe lieferte die Firma Dr. Günther Kast aus Sonthofen im Oberallgäu, die auf technische Gewebe und Spezialfasererzeugnisse spezialisiert ist. Gedruckt und gebunden wurde das Buch von der Firma Bookstation im bayerischen Anzing. Im Internet finden Sie Informationen über das gesamte Verlagsprogramm unter www.editionargus.de, zum Institut Interpretation der Hochschule der Künste Bern unter www.hkb.bfh.ch/interpretation und www.hkb-interpretation.ch. Die Deutsche Nationalbibliothek verzeichnet diese Publikation in der Deutschen Nationalbibliografie; detaillierte bibliografische Daten sind im Internet über www.dnb.de abrufbar. (C) der zeitgleich erschienenen digitalen Version: die Autorinnen und Autoren, 2019. Dieses Werk ist lizenziert unter einer Creative Commons Namensnennung-Nicht kommerziell 4.0 International Lizenz (CC BY-NC 4.0). Dor: https://doi.org/I0.26045/kp64-6I76 ISBN 978-3-93I264-92-5 